$\begin{array}{lllllllllllllllllllll}M & \text { A } & \text { T } & \text { H } & \text { E } & M & \text { A } & \text { T } & \text { I } & \text { C } & \text { A } & \text { L } & \text { E } & \text { C } & \text { O } & \text { N } & \text { O } & \text { M } & \text { I } & \text { C } & \text { S }\end{array}$

No. 10(17)

2014

\title{
ON MAXIMAL SOCIAL PREFERENCE
}

\author{
Janusz Łyko, Antoni Smoluk
}

\begin{abstract}
Mathematics and physics are based on two numbers: Archimedes' constant $\pi=3,14 \ldots$ and $e=2,71 \ldots$ - Napier's constant. The former reflects the ratio of the perimeter of a figure to its diameter and maximizes the area, given the diameter. The solutions are the disk and the circle. The latter represents the accumulated capital paid by a bank after one year from investing one unit of money at an annual interest rate of $100 \%$ under continuous compounding. The ratio of the disk's perimeter to its diameter, i.e. $\pi$, governs omnipresent cyclical motion, whereas Napier's constant determines natural growth exponential growth. Nature mixes both kinds of behaviour: there is equilibrium - vortices, and the cobweb model, dynamic growth. Our general remarks are corroborated by the theory of linear differential equations with constant coefficients. Social life - democracy and quality - despite the deceptive chaos of accidental behaviour, is also governed by a beautiful numeral law. This social number is $\lambda=2 / 3$ whose notation is derived from the Greek $\lambda \alpha o \varsigma$ meaning crowd, people, assembly. The social number, Łyko's number, is defined by the fundamental theorem. If each alternative of a maximal relation of a given profile has its frequency in this profile greater than $2 / 3$, then such relation is a group preference. This sufficient condition separates a decisional chaos from a stable economic and voting order - the preference. Also our everyday language makes use of $\lambda$. We distinguish with it upper states - elitist ones, from ordinary standards. The $2 / 3$ rule implies that in each group one third of the population prevails, while the rest are just background actors. The number $\lambda$ also appears, a bit of a surprise, in classical theorems of geometry.
\end{abstract}

Keywords: state of welfare, 2/3 rule, utylity functions, Malthus' increase, Markov's matrix.

JEL Classification: A10, C02.

DOI: $10.15611 / \mathrm{me} .2014 .10 .03$.

\section{Introduction}

The paper deals with the choice of group preference. We assume the knowledge of the preferences of each member of the group and we try to find a global preference that is binding for all members. This preference is

\footnotetext{
Janusz Lyko, Antoni Smoluk

Department of Mathematics and Cybernetics, Wrocław University of Economics, Komandorska Street 118/120, 53-345 Wrocław, Poland.

E-mail: janusz.lyko@ue.wroc.pl
} 
expected to be consistent with individual preferences in some sense. It may be a gravity centre of individual preferences or Chebyshev's centre. As the latter is the centre of the smallest circle embracing the whole set, thus we should introduce a metric in a set of preferences. Another suitable candidate to be a social preference is maximal preference. We follow that line. Generally, maximal relation is not a preference. A sufficient condition to be a preference is the $2 / 3$ rule.

In 1894, Wilhelm Windelband, who was then a famous German philosopher, put forward a distinction between nomothetic and idiographic sciences within empirical sciences, arguing that nomothetic sciences generate laws of nature, while idiographic sciences describe the manifestation of laws of nature. Obviously there are no other sciences except for empirical sciences; all of them generate laws; the application of those laws is further a domain of art. This paper recalls the $2 / 3$ rule and shows its frequent manifestations in social life and in science.

\section{Preferences}

Preferences are reflexive and transitive relations. Quality is a preference - probably the most important one. Each preference, in a sense, is a product of order and equivalence: by equalling equivalent objects, preference is changed into order. In the remainder, we will deal with linear preferences that evaluate each pair of elements from a given set of goods $X$; instead of material goods, one may think of candidates for a given position. With $K=\{0, \ldots, n\}$ denoting a finite set of $n+1$ buyers or voters, a system of preferences $\left(p_{0}, \ldots, p_{n}\right)$, where $p_{i}$ is a linear preference of the $i$ th customer, is called a profile. The elements of the product $X \times X$, i.e. the pairs of products $(x, y)$ are alternatives or decisions. If $p$ is a linear preference, then, naturally, $(x, y) \in p$ or $(y, x) \in p$, i.e. $x p y$ or $y p x$. A maximal relation $m$, defined by its profile $\left(p_{0}, \ldots, p_{n}\right)$, also denoted by $m\left(p_{0}, \ldots, p_{n}\right)$, satisfies the following condition: $x m y$ if and only if a set $\left\{i \in\{0, \ldots, n\}: y p_{i} x\right\}$ is equinumerous with a subset of $\left\{i \in\{0, \ldots, n\}: x p_{i} y\right\}$. A maximal relation is an absolute "greater than" relation. Hence, if $x m y$, then an alternative $(x, y)$ is preferred by more decision makers than $(y, x)$, unless $y m x$ also holds, then both alternatives are equivalent. A qualified majority $k$ at a 50 per cent level is a slightly different relation. If $x k y$, then an alternative $(x, y)$ is preferred by at least 50 per cent of decision makers. Relation $s$ satisfies the $2 / 3$ rule if $x s y$, and if an alternative $(x, y)$ with its profile $\left(p_{0}, \ldots, p_{n}\right)$ is preferred by a greater number - much greater - than $2 / 3$ of decision makers. Łyko's theo- 
rem states that if a relation satisfies the $2 / 3$ rule, then $m$ is a preference [ $€$ yko 2000]. A qualified majority at the $1 / 2$ level is a weaker relation. If the $2 / 3$ rule is satisfied by $m$, then it might not be satisfied by $k$, but if $k$ fulfils the $2 / 3$ rule, then naturally $m$ fulfils it as well. If all preferences in a profile are orders, then $m=k$. Generally, we distinguish two social relations $m$ and $k-$ an absolute majority $m$ and a qualified majority at a 50 per cent level. If $m$ and $k$ are preferences, then the relation $m$ is closer to an order relation than $k$. Therefore, it is a less numerous relation - fewer objects are equalized when divided by equivalence. Łyko's theorem is based on a fundamental forcing lemma.

Stochastic forcing. If the probability of an event $A$ satisfies ] $\left.\frac{2}{3}, 1\right]$ and if the probability of an event $B$ also satisfies $\left.] \frac{2}{3}, 1\right]$, then the probability of an event $A$ and $B$ satisfies $\left.] \frac{1}{3}, 1\right]$.

\section{Tertians}

A whole is an attribute, thus a property. Let small and great tertians be analogues of a median. They are quantiles partitioning a distribution into the three mentioned equally probable classes: the left-hand one with the small strength of an attribute, the middle one with normal and the right-hand one with great strength. Certainly, for the sake of convenience, we assume here a continuous distribution; as a result, each class contains $1 / 3$ of the population measured by the distribution. Such partitions may be continued, with finer subsets, more detailed and almost homogenous. A ternary division follows from natural selection and the equilibrium principle. Diversity is a sign of many influencing factors. Each quality has three levels of intensiveness, each adjective has three degrees of comparison. Tertians are fundamental and natural quantiles that divide a population into three parts (Figure 1). The $2 / 3$ rule is based on this division as it rejects medium frequencies.

\begin{tabular}{lllll} 
Badly & & Medium & & Well \\
\hline Little & $T_{0}$ & Normally & $T_{1}$ & Much
\end{tabular}

Fig. 1. Tertians - fundamental quantiles

Source: own elaboration. 


\section{The $2 / 3$ rule and its generalization}

The choice of maximal relation can be generalized. Let us take a series of sets $\left(A_{0}, \ldots, A_{n}\right)$, where set $A_{i}$ is a subset of the certain set $A$; next, let element $a \in A$. If among statements:

$$
a \in A_{0}, \ldots, a \in A_{n}
$$

there are $s$ true statements, then the frequency or occurrence of element $a$ in series $\left(A_{0}, \ldots, A_{n}\right)$ is the number

$$
f(a)=\frac{s}{n+1} .
$$

Subset $B$ of set $A$ fulfils the $2 / 3$ rule, if the following implication is true:

$$
b \in B \rightarrow f(b)>\frac{2}{3} .
$$

Hence, set $\mathrm{B}$ satisfies the $2 / 3$ rule with respect to series $\left(A_{0}, \ldots, A_{n}\right)$, if and only if each element of this set has got a frequency greater than $2 / 3$. This generalization is applied when selecting a stock portfolio, especially important and useful when a portfolio represents a certain group: it may be a marketable bank product or investment guarantee of pensions. A portfolio is a preference in a set of stocks; it can also be considered as a fuzzy subset of a stock family. A social portfolio is an algebraic sum of individual portfolios - similarly to a sum of baskets of goods. Hence, we directly arrive at classical probability, i.e. we are fully unaware of what will happen.

The alternative $(y, x)$ is a negated decision $(x, y)$; the alternative is maximal when it has a frequency greater than the frequency of negation. A maximal relation is a set of maximal decisions. Let $M$ denote a family of decisions with frequencies greater than $2 / 3$. Relation $M$ is a preference, while relation $m$ is a preference if it fulfils the $2 / 3$ rule.

Theorem. A profile satisfies the $2 / 3$ rule, i.e. maximal relation $m$ fulfils the $2 / 3$ rule with respect to this profile, if and only if the following implication holds true: if the frequency of a decision is much greater than $1 / 3$, then its frequency is much greater than $2 / 3$.

If each alternative or its negation belongs to maximal relation $m$ and if $m$ satisfies the $2 / 3$ rule, then $m$ is a linear preference. If $m$ is complete and satisfies the $2 / 3$ rule, then it is a preference. If a profile has the order type, each preference is a linear order, and $m$ satisfies the $2 / 3$ rule, then a maximal relation is a linear order. 


\section{Constant $\lambda$}

The constant $\lambda=\frac{2}{3}$ and also its inverse 1.5, i.e. one and a half, appear in ordinary language; they approximately describe statistical populations, dealing with typical proportions. In everyday language, in journalism and scientific papers, $2 / 3$ refer to a central mass, the kernel of the population. A statement similar to: nearly two thirds of typical companies have some type of balanced scorecard program in place [Hackett 2004], represents a universal standard. Only $1 / 3$ of cases of mentally disordered persons obsessed by evil require exorcism [Szczepański 2013]. The damaged painting: A scuffle of peasants with pilgrims, has a missing horizontal wedge reaching $2 / 3$ of the width of the picture [Steinborn 2006, p. 68]. We make decisions influenced by various criteria. An attorney's office typically throws out $2 / 3$ of cases [Giertych 2013]. Only $1 / 3$ of cases in a given institution are valuable, i.e. they generate income, while the remaining $2 / 3$ are not worthwhile. Money rules the world and wealthy people are not more than $1 / 3$ of the population.

The number $2 / 3$ has become familiar in our everyday language due to purely empirical influences, still it is based on a strong theory - Łyko's theorem. It is actually a border between two worlds: the world of order and the world of chaos. Its role in social sciences is the same as that of $\pi$ and $e$ in physics and biology. It represents a simple and beautiful law of nature just like $\pi$ governs cyclicality and $e$ runs natural growth. We all make mistakes; $2 / 3$ of business decisions are wrong - it is difficult to guess the future, even more difficult to guess are the forthcoming preferences of the entire society [Tracy, Kozak 2011, p. 52].

Doctors believe that $2 / 3$ of Poles suffer from periodontitis, while the elite or $1 / 3$ of the population are healthy [Reklama 2014]. Medical surveys provide evidence that $1 / 3$ of those diagnosed with Alzheimer's disease - brain cell failure and dementia - would have escaped the disorder if only they would had been living a healthy life. Measures preventing Alzheimer's disease are physical and intellectual activity, healthy food, avoiding tobacco, a happy life. Nevertheless, $2 / 3$ of those diagnosed have been wronged in some way by fate: regardless of their lifestyle, they contracted Alzheimer's. An elite in the population of ill people is made up of $1 / 3$ of accidental individuals who might have avoided the disease if they had lived a different life - a more prudent life [AS 2014]. The disease is seen as likely in $1 / 3$ of the population, while as unavoidable in $2 / 3$. Regarding a bipolar disorder, $1 / 3$ of those affected during periods of depression seek death to alleviate the soul's pain. 
Although energy efficient houses are more costly by 20 per cent, still $2 / 3$ of the population believe they are future solution [Olechowski 2014]. People in Poland are reluctant savers and investors. We are a poor society and the low propensity to save makes us relatively poorer. It turns out that $2 / 3$ of households have no savings at all and only $1 / 3$ are managed rationally [Koprowicz 2014]. Ten years after EU accession, Poland's GDP has reached $2 / 3$ of the average income of the richest seven EU countries.

Nobel prize laureates in physics, chemistry, medicine and economics nominated by the Swedish Academy of Sciences have been increasingly U.S. scholars. In 2002, Americans absolutely outnumbered the other Nobel prize recipients, with their share approaching $2 / 3$. It is a snowball effect; what counts is money allocated to research, freedom of innovation, brain drain from the rest of the world, and, naturally, the colleagues who have been awarded the prize in the past. Thus, Americans are the elite of researchers [The Sutton Trust 2003]. Published research outcomes, especially those making headlines, are mostly regarded as half-truths at best. Medical sciences are implicated here [Gawrońska 2014]. 'Mostly' means of course a fraction close to $2 / 3$. Research conducted under pressure of the health industry are conducted more and more rapidly and cheaply, with data adjusted so as to get a predetermined result. One group of scholars proves that a given substance is beneficial, while another demonstrates that it is detrimental or completely irrelevant. The assertion depends on the sponsor's will; in the end, 15 million scientists deserve to be employed. Science drives steps forward; there is one scientist per each 500 inhabitants of the globe, 2 per thousand of the population are employed in science.

In Poland, $1 / 3$ of secondary school graduates failed examinations for a final certificate in 2004 [Dębek 2014]. The mass media made a noise, although there are no reasons to lament. The result indicates that the examinations were conducted correctly, since $1 / 3$ of the weakest failed consistently with the distribution of abilities. It is time to stop thinking that the "maturity diploma' (matura) should be awarded to everyone, as only a few high jumpers are able to clear $1.50 \mathrm{~m}$ and only a few chess players become masters. Thomas Rehdiger (1540-1576), a humanist and a collector from Wrocław, amassed nearly sixty portraits of famous contemporary persons. Until the present day twenty paintings have survived, including four portraits of women. Time has destroyed $2 / 3$ of the collection. Obviously, the most famous, or the elite, represent $1 / 3$ of the upper class. It turns out that almost $1 / 3$ of Rehdiger's top class are women; the major part of the elite is male. Therefore, the gentle sex is the elite of the elite. Human life can be divided 
into three periods: adolescence up to age of 25 years, mature age between 25 and 50 years, and old age between 51 and 75 years. Youth is a period of development, middle age is represented by stability, and old age embraces recession including decay; thus, we like $2 / 3$ of our lives, while $1 / 3$ is rather disturbing. It turns out that $1 / 3$ of papers presented during Southern Poland Conferences are purely mathematical, while $2 / 3$ involve statistics and econometrics.

\section{Approximations}

The $2 / 3$ rule implies a day's division into three equal sequences and a long-desired eight hour work day: 8 hours work, 8 hours relaxation, 8 hours sleep. The number $2 / 3$ defines the centre of gravity on line segment $\mathrm{AB}$, when we place the following masses on pan $\mathrm{A}$ :

$$
\frac{1}{2}+\frac{1}{8}+\frac{1}{32}+\ldots=\frac{2}{3}
$$

and following masses on pan B:

$$
\frac{1}{4}+\frac{1}{16}+\frac{1}{64}+\ldots=\frac{1}{3} .
$$

Pan A is distant from the centre of gravity by one unit and houses $2 / 3$ of the load, while pan B is distant from the centre of gravity by two units and houses $1 / 3$ of the mass. It provides an insight into the structure of the solution to the recurrence equation

$$
x_{n+2}=\frac{1}{2}\left(x_{n}+x_{n+1}\right) .
$$

Wine experts advise on how to maintain a rational private wine cellar [Brzeziński 2013]. The key principle of such a collection is the dichotomy into cheap liquors for daily consumption and more expensive wines that increase their value as they mature. Cheap wines should represent $2 / 3$ of the collection. The French scale of champagne as regards sweetness is defined by the arithmetic sequence:

$$
a_{0}=0, a_{n+1}=a_{n}+\frac{3}{2}, \quad n \in N .
$$

Brut means 0\% sugar, i.e. $a_{0}$; extra sec is $a_{1}=1.5 \%$ sugar; sec is $a_{2}=3 \%$; demi-sec is $a_{3}=4.5 \%$; demi-doux is $a_{4}=6 \%$ sugar; doux is $a_{5}=7.5 \%$ sugar. Łyko's number $\lambda=\frac{2}{3}$ and its reciprocal 3/2 are Man's helpful companions. It is a law of nature that is so convenient as the wheel. Adonis used to spend 
$1 / 3$ of the year in Hades and the remaining $2 / 3$ among humans living on earth. Two thirds of the area of Honsiu, the biggest Japanese island, are forest; $2 / 3$ of Poles aged 30 and more are overweight. The heights of an equilateral triangle intersect in the centre of gravity that cuts them in the ratio 1:2. The shorter line segment is the radius of an inscribed circle, while the longer one - the radius of an circumscribed circle of the triangle (Figure 2). The number $\lambda$ either defines a two-point distribution $(1 / 3,2 / 3)$ or it reflects geometric orderliness - a coefficient of harmony.

\section{Geometry and $\lambda$}

The volume $V_{0}$ for a cube of edge length $a$ is given by the simple formula $V_{0}=a^{3}$; the volume $V_{1}$ of the inscribed cylinder is a fraction of volume $V_{0}$ or $V_{1}=\frac{\pi}{4} a^{3}$; the volume $V_{2}$ of the ball inscribed in a cube and in a cylinder is a twofold fraction: of the volume of a cube, or $V_{2}=\frac{\pi}{6} a^{3}$, and of the volume of a cylinder, or $V_{2}=\frac{2}{3} V_{1}$. The volume of the segment of a ball a wedge produced by planes intersecting a ball in its poles and forming angle $\alpha$-depends on the radius of ball $r$ and arc $\alpha$, or $V=\lambda \alpha r^{3}$. If $\alpha=2 \pi$, then $V$ is the volume of a ball, and $V=2 \lambda \pi r^{3}$. The number $\lambda$ in those formulas competes with Archimedes' constant.

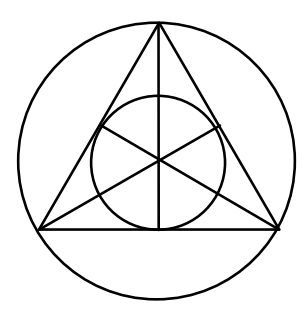

Fig. 2. The $2 / 3$ rule

Source: own elaboration.

A cuboid container with a rectangular face is to be formed from square steel sheets with a given edge length $a \in R$; naturally, $a>0$. Technology requires to cut out the same square in each corner of the sheet; the remainder is folded and welded to make the container (Figure 3). What squares 
should be cut out so that the volume of the container is maximal? It turns out that the edge of the square should be $1 / 6$ of the initial sheet edge; the loss is $1 / 3$ of the length of the edge.

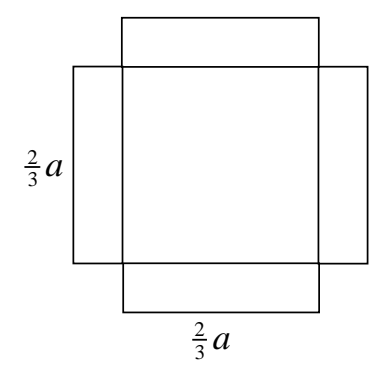

Fig. 3. Optimal dissection

Source: own elaboration.

\section{History of finance}

The $2 / 3$ rule is also present when we study the history of finance. The simple interest rate of $2 / 3 \%$ per month implies $8 \%$ per year. Such was the customary interest rate resulting from the economic efficiency in ancient Rome. Is Professor Dobija not rediscovering an ancient formula when he is promoting $8 \%$ today? The calculation of alternatives' frequency in profiles of preferences to establish an equilibrium price of capital is perhaps of the same nature - activity has to be effective and efficient.

A theatre auditorium - representing modern democratic equality perceived by marketers - is divided into two parts: the greater one, with standing room for the poor, and the smaller one, with seats for the rich. The poor received approximately $2 / 3$ of the area [Banasiak 2013]. The number $\lambda$ governs the quality and the wealth in a similar fashion as $\pi$ governs the area and the volume of a ball. The maximal area - hence, a circle and $\pi$, the stable majority - thus, preferences and $\lambda$. A third of adult Americans are extremely obese today [Milewski 2013]. The bes was an ancient Roman coin valued at two-thirds of an as, i.e. as an as made 12 unciae, the value of the bes was 8 unciae. The main heir - heres ex besse - takes $2 / 3$ of the wealth. A testator named his wife as the heir of $1 / 3$ of the property in case she gave birth to a son and $2 / 3$ of the property if a girl was born, with the residue passing on to the child. Now, twins were born - a boy and a girl. How to divide the inheritance according to the father's will? Roman lawyers enjoyed solving such a problem. Their solution followed the axiom declaring that the son was to 
receive twice as much as his mother, and the mother twice as much as her daughter. Let $x$ denote the son's share, $y$-the mother's share and $z$-the daughter's share, then certainly $x+y+z=1$ subject to $x=2 y, y=2 z$. It follows that the son receives $4 / 7$, the mother $2 / 7$ and the daughter $1 / 7$ of the inheritance. Such a distribution seems unfair, because it harms the mother. In any case, the testator meant at least $1 / 3$ of the wealth passing on to her. The will defined two 3-point distributions: $\left(\frac{2}{3}, \frac{1}{3}, 0\right)$, if a son is born, and $\left(0, \frac{2}{3}, \frac{1}{3}\right)$, if a daughter is born. Both equally probable events occurred, therefore the solution is provided by a simple mean - a convex combination of end points; it is a point $\left(\frac{1}{3}, \frac{1}{2}, \frac{1}{6}\right)$ indicating that the son gets $1 / 3$, the wife $1 / 2$, and the daughter $1 / 6$ of the inheritance. A convex division is equally logical and fair - the mother is rewarded. A simple average - as above mentioned difference equation demonstrates - results in the $2 / 3$ rule that was followed by the testator. In addition, we see that 6 is a perfect number, because it is a sum of its proper divisors: $6=1+2+3$.

\section{Theatre and voting}

A theatre must make a profit to exist. The repertory of Krystyna Janda's theatre includes 60 plays, but only ten are income-producing and they support the remainder [Janda 2013]. The middle class is formed by $1 / 3$ of society, while the upper middle class supporting the theatre is half of the middle class, i.e. $1 / 6$. The numbers $\frac{1}{6}, \frac{1}{3}, \frac{1}{2}$ depend on $\lambda$ just like fractions: $\frac{\pi}{6}, \frac{\pi}{3}$, $\frac{\pi}{2}$ depend on $\pi, 25 \%$ of $\lambda$ equal precisely $\frac{1}{6}, 50 \%$ - equal $\frac{1}{3}$, and $75 \%-$ equal $\frac{1}{2}$. Prices of jewellery with diamonds also reveal a three-point distribution: $\left(\frac{1}{6}, \frac{1}{3}, \frac{1}{2}\right) ; \frac{1}{6}$ of the price equals the value of the unprocessed gem, $\frac{1}{3}$ - cost of work and additional materials, half of the price is generated by marketing [Info Grafika 2013]. A similar structure of prices is typical for cosmetics, especially among top brands. The best blended whisky represents a distribution $\left(\frac{1}{3}, \frac{2}{3}\right)$, i.e. a blend of $1 / 3$ of barley whisky and $2 / 3$ of corn whisky. The same two-point distribution is represented in the Polish mead 'Półtorak' ('one-and-a-half'): one unit of water and two units of honey. The taste buds on our tongues are therefore able to detect $2 / 3$; it is a boundary of quality - the point contains an optimum, an equilibrium. 
A three-point distribution $v=\left(v_{0}, v_{1}, v_{2}\right)$, where, naturally, $v_{0}+v_{1}+v_{2}=1$ and $v_{i} \in[0,1]$, represents voting; votes in favour are $v_{0}$, neutral (abstaining) are $v_{1}$, and votes against are $v_{2}$. Voting by a simple majority is defined by $v_{0}>v_{2}$, and with absolute majority by $v_{0}>0.5$. Let $q \in[0,1]$; a simple majority $v$ is called a qualified majority at level $q$ when $v_{0} \geq q$. A qualified majority at a 0.5 level is not necessarily an absolute majority. If $q=0$, then a qualified majority is merely a simple majority. The most important cases are voted on by a qualified majority at level $\lambda$. Why? One of the criteria behind the choice of $q$ is that of stability. Reaching agreement of opinions at a level $\lambda$ assures the long-term decisional efficiency of the governing body, due to the invariant time series - as a result, there are no problems with absolute majority.

\section{More on difference equations}

Let us take any two points $M_{0}$ and $M_{1}$ in Banach space $E$. If $p, q \in[0,1]$ and $p+q=1$, then a sequence defined by a difference equation

$$
M_{n+2}=p M_{n}+q M_{n+1}, n \in N,
$$

is an oscillating sequence $\left(M_{0}, M_{1}, M_{0}, M_{1}, \ldots\right)$ when $p=1$, thus, it is convergent only if $M_{0}=M_{1}$. If $p<1$, then this sequence converges to a point

$$
M=\frac{p}{1+p} M_{0}+\frac{1}{1+p} M_{1} .
$$

To make it evident, it suffices to examine an ordinary difference equation

$$
x_{n+2}=p x_{n}+q x_{n+1}, \text { where } x_{n} \in R .
$$

Its characteristic equation $\sigma^{2}-q \sigma-p=0$ has two roots $\sigma_{1}=1, \sigma_{2}=-p$, thus the general solution is given by the formula

$$
x_{n}=c_{1} \sigma_{1}^{n}+c_{2} \sigma_{2}^{n}, n \in N,
$$

with constants $c_{1}$ and $c_{2}$ determined so that initial conditions are fulfilled [Smoluk 2007]. Certainly, the sequence $\left(x_{n}\right)$ is convergent and $\lim \left(x_{n}\right)=c_{1}$. Since

thus

$$
\begin{gathered}
c_{1}+c_{2}=x_{0}, \\
c_{1}-p c_{2}=x_{1},
\end{gathered}
$$

$$
c_{1}=\frac{p}{1+p} x_{0}+\frac{1}{1+p} x_{1}
$$


If $p=\frac{1}{2}$, then we get a remarkable equality: $M=\frac{1}{3} M_{0}+\frac{2}{3} M_{1}$. Subsequent arithmetic means $M_{n+2}=\frac{1}{2}\left(M_{n}+M_{n+1}\right)$ lead to a prudent forecasting rule: assign a weight $\frac{1}{3}$ to the past, and twice as much, or $\lambda$, to the present age.

Ordinary difference equations that define a sequence $\left(x_{n}\right)$ are generated by a linear operator $A: R^{2} \rightarrow R^{2}$ given by matrix

$$
A=\left(\begin{array}{ll}
0 & 1 \\
p & q
\end{array}\right),
$$

whereas an equation in a Banach space is generated by the operator $B: R^{4} \rightarrow R^{4}$ given by matrix

$$
B=\left(\begin{array}{llll}
0 & 0 & 1 & 0 \\
0 & 0 & 0 & 1 \\
p & 0 & q & 0 \\
0 & p & 0 & q
\end{array}\right) .
$$

Space $R^{4}$ is not accidental here; two points in a plane are defined by four coordinates. Matrix $B$ is obtained from $A$ making reference to an isomorphism between the field of diagonal matrices

$$
\left\{\left(\begin{array}{ll}
x & 0 \\
0 & x
\end{array}\right) \in M_{2}(R): x \in R\right\}
$$

and the field of real numbers $R$. Since

$$
C B C^{-1}=\left(\begin{array}{cccc}
0 & 1 & 0 & 0 \\
p & q & 0 & 0 \\
0 & 0 & 0 & 1 \\
0 & 0 & p & q
\end{array}\right),
$$

where

$$
C=\left(\begin{array}{llll}
1 & 0 & 0 & 0 \\
0 & 0 & 1 & 0 \\
0 & 1 & 0 & 0 \\
0 & 0 & 0 & 1
\end{array}\right),
$$


hence $\operatorname{det}(B-\sigma I)=\operatorname{det}^{2}(A-\sigma)=(\sigma-1)^{2}(\sigma+p)^{2}$. Thus sequence $\left(B^{n}\right)$, with $p<1$, has a limit

$$
\left(\begin{array}{cccc}
\frac{p}{1+p} & 0 & \frac{1}{1+p} & 0 \\
0 & \frac{p}{1+p} & 0 & \frac{1}{1+p} \\
\frac{p}{1+p} & 0 & \frac{1}{1+p} & 0 \\
0 & \frac{p}{1+p} & 0 & \frac{1}{1+p}
\end{array}\right)
$$

\section{The stock exchange, $\lambda$ and Markov chains}

There have been attempts to explain a stock exchange by Markov processes. A stock exchange is a random walk with three states: low stock prices of bad companies; stock prices of medium companies fluctuating around the market value; rising stock prices of good companies. Such a categorization of listed companies underlies recommendations of market analysts to investors: sell - decline of value is looming, hold - stable prices, buy - increase of value is expected. The experience shows that transitioning from a given state to a new state depends only on a current moment, and does not depend on a more distant past, thus a stock exchange is a simple example of a Markov chain. The transition matrix

$$
T=\left(\begin{array}{ccc}
\frac{2}{3} & \frac{1}{3} & 0 \\
\frac{1}{6} & \frac{2}{3} & \frac{1}{6} \\
0 & \frac{1}{3} & \frac{2}{3}
\end{array}\right)
$$

exemplifies a rational model of a stock exchange. The rows and columns correspond to the states: bad $-B$, medium $-M$ and good $-G$, respectively. The first row of matrix $T$ includes the probabilities of transitioning from state $B$ to states $B, M$ and $G$; the second - probabilities of transitioning from state $M$, and the third - from state $G$ also to states $B, M$ and $G$. Positive probabilities are only those of transitioning from state $M$. Transitioning in one step from state $B$ to state $G$, and back, from state $G$ to state $B$ are not possible. Matrix $T$ has three eigenvalues: $\sigma_{1}=\frac{1}{3}, \sigma_{2}=\frac{2}{3}, \sigma_{3}=1$. Eigenvalue $\sigma_{1}$ corresponds to eigenvector $a_{1}=(1,-1,1)$, eigenvalue $\sigma_{2}-$ to eigenvector $a_{2}=(1,0,-1)$, and eigenvalue $\sigma_{3}-$ to eigenvector $a_{3}=(1,1,1)$. The matrix 


$$
A=\left(\begin{array}{ccc}
1 & 1 & 1 \\
-1 & 0 & 1 \\
1 & -1 & 1
\end{array}\right)
$$

transforms the natural basis: $e_{1}=(1,0,0), e_{2}=(0,1,0)$ and $e_{3}=(0,0,1)$ into a basis composed of eigenvectors: $a_{1}, a_{2}, a_{3}$. In a new basis matrix $T$ will be a diagonal matrix $S=A^{-1} T A$, where

$$
A^{-1}=\left(\begin{array}{ccc}
\frac{1}{4} & -\frac{1}{2} & \frac{1}{4} \\
\frac{1}{2} & 0 & -\frac{1}{2} \\
\frac{1}{4} & \frac{1}{2} & \frac{1}{4}
\end{array}\right), \quad S=\left(\begin{array}{ccc}
\frac{1}{3} & 0 & 0 \\
0 & \frac{2}{3} & 0 \\
0 & 0 & 1
\end{array}\right) .
$$

Matrix $S$ has no probabilistic interpretation, or it is not equivalent to the Markov matrix $T$. Nevertheless, matrix $S$ is enormously useful, as it significantly facilitates the computation of the boundary matrix of sequence $\left(T^{n}\right)$. Sequence $\left(S^{n}\right)$ has a limit, of course, and

$$
\lim \left(S^{n}\right)=\left(\begin{array}{lll}
0 & 0 & 0 \\
0 & 0 & 0 \\
0 & 0 & 1
\end{array}\right),
$$

and since $T^{n}=A S^{n} A^{-1}$, hence $B=\lim \left(T^{n}\right)=A \lim \left(S^{n}\right) A^{-1}$. Finally, we get

$$
B=\left(\begin{array}{ccc}
\frac{1}{4} & \frac{1}{2} & \frac{1}{4} \\
\frac{1}{4} & \frac{1}{2} & \frac{1}{4} \\
\frac{1}{4} & \frac{1}{2} & \frac{1}{4}
\end{array}\right) .
$$

The result implies that the medium state is asymptotically privileged - it contains $50 \%$ of companies, whereas the two other states embrace $25 \%$ of companies each. The golden mean also governs a stock exchange.

Undeniably, alle Theorie ist grau - i.e. all theories are grey, but the laws of science are clear - they shine a light of the inner truth. One law of science is the $2 / 3$ rule. The qualified majority of $2 / 3$ is not an arbitrary boundary, one of many possible values, let us emphasize it once more - it is a principle that distinguishes a social preference. A natural definition of number $2 / 3$ is similar to that of number $\pi, e$ and other fundamental constants of physics. A majority relation is always a preference when each alternative in a profile has the frequency strongly greater than $2 / 3$. 
The Markov matrix

$$
M=\left(\begin{array}{ccc}
\frac{2}{3} & \frac{1}{3} & 0 \\
\frac{1}{3} & \frac{1}{3} & \frac{1}{3} \\
0 & \frac{1}{3} & \frac{2}{3}
\end{array}\right)
$$

is obtained from matrix $T$ by slightly modifying the second row. The minor quantitative correction leads to a qualitative change of the process. Arguably, $M$, due to its symmetry, better embodies the nature of stock exchange behaviour than $T$. A spectrum of matrix $M$ is the set containing three elements,

$$
\operatorname{sp} M=\left\{0, \frac{2}{3}, 1\right\},
$$

whereas the asymptotic matrix $\lim \left(M^{n}\right)$, obtained analogously as for matrix $T$, has all entries equal. Transitioning from each state to any other state has the same probability; there are equal numbers of companies in each state $(1 / 3)$. No matter what the initial position was, after sufficiently long walking, one can arrive anywhere. Thus, it is a maximal entropy - we live in a world of uncertainty: I am on top today, but tomorrow I may fall. Precisely this philosophical conclusion derived from model $M$ makes it almost a law of science - a law of universal equality and justice, a law of equilibrium. There are no privileged, the condition of everyone is equally uncertain: an unbiased fate rules the world. The boundary states $B$ and $G$ are better off only at the beginning - they attract more strongly. Finally, we have democracy there is no poverty, there is no luxury either. The wheels of fortune roll on. Naturally, some Markov matrices $U$ have no boundary matrix $\lim \left(U^{n}\right)$. If the eigenvalue of a Markov matrix is a complex number with modulus equal 1 or greater, then there exists no boundary matrix.

Remark. If $\alpha^{2}+\beta^{2}=1$, then a complex matrix

$$
\left(\begin{array}{cc}
\alpha-\beta i & 0 \\
0 & \alpha+\beta i
\end{array}\right)
$$

is equivalent over a field of complex numbers to a real matrix

$$
\left(\begin{array}{cc}
\alpha & -\beta \\
\beta & \alpha
\end{array}\right)
$$

representing a rotation of a plane. Superpositions of rotations do not stabilize, except for equivalence. 


\section{Descartes and social choice}

The folium of Descartes

$$
x^{3}+y^{3}=3 a x y
$$

is a third-degree algebraic curve with strongly positive parameter $a$. A rotation by an angle $\frac{\pi}{4}$, i.e. a transformation:

$$
\begin{aligned}
& x=\frac{1}{\sqrt{2}} x^{\prime}-\frac{1}{\sqrt{2}} y^{\prime}, \\
& y=\frac{1}{\sqrt{2}} x^{\prime}+\frac{1}{\sqrt{2}} y^{\prime},
\end{aligned}
$$

yields a new form of this curve - the coordinates are denoted as before for the sake of convenience

$$
x^{3}+3 x y^{2}=3 \rho\left(x^{2}-y^{2}\right), \text { where } \rho=\frac{a}{\sqrt{2}} .
$$

Since

$$
y^{2}=x^{2} \frac{3 \rho-x}{3 \rho+3 x},
$$

the folium of Descartes has two branches:

and

$$
f(x)=x \sqrt{\frac{3 \rho-x}{3 \rho+3 x}}
$$

$$
g(x)=-x \sqrt{\frac{3 \rho-x}{3 \rho+3 x}},
$$

where $-\rho<x \leq 3 \rho$. A straight line $x=-\rho$ is the asymptote. To calculate a primitive function $F$ of function $f$, we substitute

$$
t^{2}=\frac{3 \rho-x}{\rho+x}
$$

then the function

$$
F(x)=\int f(x) d x
$$

becomes

$$
H(t)=-\frac{4}{\sqrt{3}} a^{2} \int \frac{3 t^{2}-t^{4}}{\left(1+t^{2}\right)^{3}} d t
$$


The integrand is the division of even polynomials. It is an even function of a special form, that can be written as a sum of three simple even fractions

$$
\frac{A}{1+t^{2}}+\frac{B}{\left(1+t^{2}\right)^{2}}+\frac{C}{\left(1+t^{2}\right)^{3}}=\frac{3 t^{2}-t^{4}}{\left(1+t^{2}\right)^{3}} \text {. }
$$

This observation facilitates computations, because it suffices to determine just three unknowns, otherwise, six unknowns are to be found. Hence, we have a system of equations:

$$
\begin{gathered}
A+B+C=0, \\
2 A+B=3, \\
A=-1,
\end{gathered}
$$

whose solution is given by a vector with elements: $A=-1, B=5, C=-4$.

Lemma. If a degree of an even polynomial $p$ does not exceed $2 n$, then a rational function

$$
r(x)=\frac{p(x)}{\left(1+x^{2}\right)^{n}}
$$

is a linear combination of simple even fractions, i.e.

$$
r=\sum_{k=0}^{n} A_{k} a_{k},
$$

where

$$
a_{k}(x)=\frac{1}{\left(1+x^{2}\right)^{k}} .
$$

For the sake of editorial simplicity, a constant function is considered as a simple even fraction.

However, if a degree of an even polynomial $q$ does not exceed $2 n+2$, then a rational function

$$
s(x)=\frac{q(x)}{\left(1+x^{2}\right)^{n+1}}
$$

is a linear combination of simple odd fractions, i.e.

$$
s=\sum_{k=0}^{n} B_{k} b_{k}
$$

where

$$
b_{k}(x)=\frac{x}{\left(1+x^{2}\right)^{k+1}}
$$


The lemma is corroborated by identities:

$$
\begin{gathered}
x^{2} a_{n+1}(x)=a_{n}(x)-a_{n+1}(x), \\
x^{2} b_{n+1}(x)=b_{n}(x)-b_{n+1}(x)
\end{gathered}
$$

and, naturally, by the principle of induction. The coefficients $A_{k}$ and $B_{k}$ are obtained without solving the equations.

Let

$$
I_{n}(t)=\int \frac{1}{\left(1+t^{2}\right)^{n+1}} d t, n \in N,
$$

then the calculations are simplified by recursion:

$$
I_{0}(t)=\operatorname{arctg} t
$$

$$
I_{n+1}(t)=\frac{2 n+1}{2 n+2} I_{n}(t)+\frac{1}{2 n+2} \frac{t}{\left(1+t^{2}\right)^{n+1}}, n \in N .
$$

As a result, we get

$$
I_{1}(t)=\frac{1}{2} \operatorname{arctg} t+\frac{1}{2} \frac{t}{1+t^{2}}
$$

and

$$
I_{2}(t)=\frac{3}{8} \operatorname{arctg} t+\frac{3}{8} \frac{t}{1+t^{2}}+\frac{1}{4} \frac{t}{\left(1+t^{2}\right)^{2}}
$$

or

$$
H(t)=-\frac{4}{\sqrt{3}} a^{2} \frac{t^{3}}{\left(1+t^{2}\right)^{2}} .
$$

Recurring to variable $x$, we obtain the primitive function $F(x)=H(t(x))$, where obviously

$$
t(x)=\sqrt{\frac{3 \rho-x}{\rho+x}} .
$$

Since: $\quad t(-\rho)=\infty, \quad t(0)=\sqrt{3}, \quad t(3 \rho)=0, \quad$ thus: $\quad F(-\rho)=H(\infty)=0$, $F(0)=H(\sqrt{3})=-\frac{3}{2} \rho^{2}, F(3 \rho)=H(0)=0$. This implies that the area $3 \rho^{2}$ adjacent to the asymptote is equal to the area of the loop (Figure 4); hence, each of them measures $\frac{3}{2} a^{2}$. 


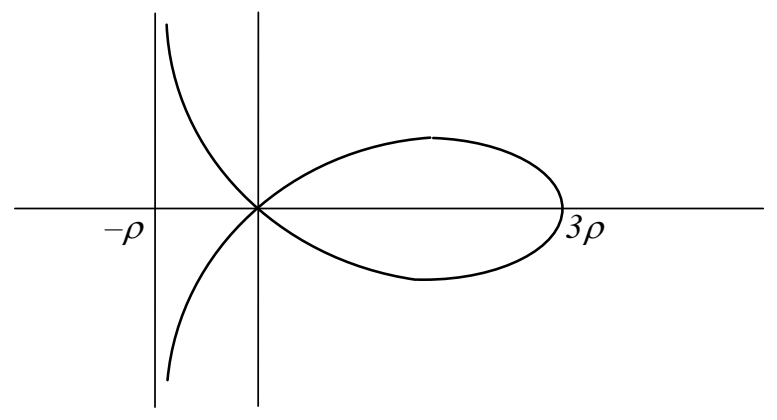

Fig. 4. The folium of Descartes

Source: own elaboration.

We will also determine curvature $\kappa(f, 0)$ of function $f$ at zero. Since

$$
\kappa(f, x)=\frac{f^{\prime \prime}(x)}{\left(1+\left(f^{\prime}(x)\right)^{2}\right)^{\frac{3}{2}}},
$$

hence, for calculating the curvature at zero, we need to solve for $f^{\prime}(0)$ and $f^{\prime \prime}(0)$. It holds:

$$
f^{\prime}(x)=\left(\frac{3 \rho-x}{3 \rho+3 x}\right)^{\frac{1}{2}}-\frac{6 \rho x}{(3 \rho+3 x)^{2}}\left(\frac{3 \rho-x}{3 \rho+3 x}\right)^{-\frac{1}{2}},
$$

thus $f^{\prime}(0)=1$. Next, we have

$$
f^{\prime \prime}(x)=-\frac{12 \rho}{(3 \rho+3 x)^{2}}\left(\frac{3 \rho-x}{3 \rho+3 x}\right)^{-\frac{1}{2}}+x h(x),
$$

where function $h$ has an intricate form; we ignore its explicit writing as it does not influence the value of the second derivative at zero. Hence, it holds $f^{\prime \prime}(0)=-\frac{4}{3 \rho}$, therefore,

$$
\kappa(f, 0)=-\lambda \frac{1}{a} .
$$

Function $f$ is a convex branch of the folium of Descartes, so its curvature is negative. Radius $r$ of a tangent circle is the reciprocal of the curvature; it can be considered as a positive number. We obtain therefore

$$
r=\frac{3}{2} a \text {. }
$$


We can see that the number $\lambda$ governs the folium of Descartes in a similar way to $\pi$ governing a circle. Although it is not an accidental resemblance, still its natural interpretation has not been available as of this time of writing. Archimedes' constant can be determined empirically, also Łyko's number can be determined empirically in two ways: where it separates chaos from order, we calculate frequencies, while in geometry, we measure the folium of Descartes. Namely, if $a=1$, then the curvature at zero of both branches of the folium equals $\lambda$, disregarding the signs.

\section{References}

AS (2014). Ucz się, będziesz zdrowy. Angora 35. 24 August. P. 73.

Banasiak J. (2013). Trzy garnki za 6000 zł. Angora 39. 29 September. P. 55.

Brzeziński M. (2013). Rentrée. Angora 38. 22 September. P. 53.

Dębek K. (2014). Pomyst. Gazeta Południowa. 9 July. P. 2.

Gawrońska M. (2014). Jaś Fasola na tropie. Gazeta Wyborcza. 5 September. Pp. 26-27.

Giertych R. (2013). Ja do polityków z ofertami nie dzwonię. Angora 21. 26 May. P. 14.

Info Grafika (2013). Inwestycje alternatywne. Kurier 3. Uniwersytet Ekonomiczny w Krakowie. P. 79.

Hackett (2004). Balanced Scorecards: Are Their 15 Minutes of Fame Over? Internet.

Janda K. (2013). Ja nie zwolnię, bo tak lubię. Gazeta na Wigilię. Dodatek do Gazety Wyborczej. 24 December. Pp. 4-6.

Koprowicz C. (2014). Sztuka oszczędzania. Newsweek Polska. 30 June. Pp. 77-79.

Łyko J. (2000). Twierdzenia Arrowa a ordynacje. In: A. Smoluk (ed.). Elementy metrologii ekonomicznej. Wydawnictwo Akademii Ekonomicznej we Wrocławiu. Pp. 165-168.

Milewski P. (2013). Zawodnik wagi ciężkiej. Newsweek Polska. 18 November. Pp. 82-84.

Olechowski J. (2014). Gra w zielone. Newsweek Polska. 15 September. Pp. 78-80.

Reklama (2014). Zapalenia przyzębia - paradontoza. Angora 35. 24 August. P. 31.

Smoluk A. (2007). Podstawy analizy matematycznej. Wydawnictwo Akademii Ekonomicznej we Wrocławiu.

Steinborn B. (2006). Katalog zbiorów malarstwa niderlandzkiego. Muzeum Narodowe we Wrocławiu.

Szczepański J. (2013). Egzorcyści wracają do cienia. Gazeta Wrocławska. 6 December. P. 23.

The Sutton Trust (2003). Analysis of Nobel Prizes. Internet.

Tracy B., Kozak R. (2011). Wędrówki z Gandalfem. MT Biznes. Warszawa. 